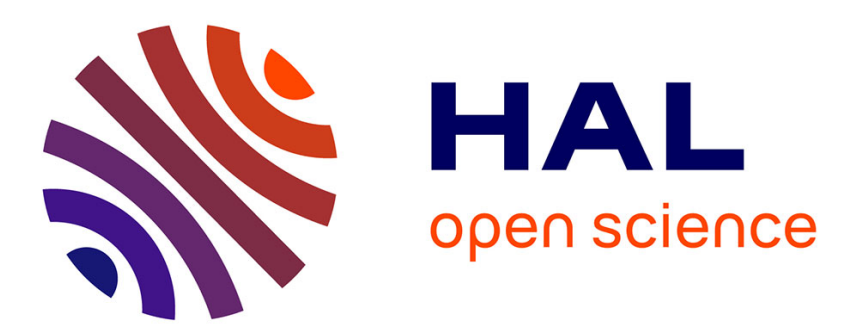

\title{
Simultaneous Bifurcation Diagrams of Carrier Number and Optical Intensity of External Cavity Laser
}

\author{
C. y Chang, Daeyoung Choi, A. Locquet, Michael J Wishon, K. Merghem,
} Abderrahim Ramdanem, François Lelarge, A. Martinez, D. S Citrin

\section{- To cite this version:}

C. y Chang, Daeyoung Choi, A. Locquet, Michael J Wishon, K. Merghem, et al.. Simultaneous Bifurcation Diagrams of Carrier Number and Optical Intensity of External Cavity Laser. CLEO: Applications and Technology, 2016, San Jose, United States. pp.JW2A.72, 10.1364/CLEO_AT.2016.JW2A.72 . hal-03085194

\author{
HAL Id: hal-03085194 \\ https://hal.science/hal-03085194
}

Submitted on 21 Dec 2020

HAL is a multi-disciplinary open access archive for the deposit and dissemination of scientific research documents, whether they are published or not. The documents may come from teaching and research institutions in France or abroad, or from public or private research centers.
L'archive ouverte pluridisciplinaire HAL, est destinée au dépôt et à la diffusion de documents scientifiques de niveau recherche, publiés ou non, émanant des établissements d'enseignement et de recherche français ou étrangers, des laboratoires publics ou privés. 


\title{
Simultaneous Bifurcation Diagrams of Carrier Number and Optical Intensity of External Cavity Laser
}

\author{
C. Y. Chang ${ }^{1,2}$, Daeyoung Choi ${ }^{2,3}$, A. Locquet ${ }^{2,3}$, Michael J. Wishon ${ }^{2,3}$, K. Merghem ${ }^{4}$, \\ Abderrahim Ramdanem ${ }^{4}$, François Lelarge ${ }^{4,5}$, A. Martinez ${ }^{4}$ and D. S. Citrin ${ }^{2,3}$ \\ ${ }^{1}$ School of Physics, Georgia Institute of Technology, Atlanta, Georgia 30332-0430, USA. \\ ${ }^{2}$ UMI 2958 Georgia Tech-CNRS, Georgia Tech Lorraine, 2 Rue Marconi F-57070, Metz, France. \\ ${ }^{3}$ School of Electrical and Computer Engineering, Georgia Institute of Technology, Atlanta, Georgia 30332-0250, \\ USA. \\ ${ }^{4}$ CNRS, Laboratory for Photonics and Nanostructures, Route de Nozay, 91460 Marcoussis, France \\ ${ }^{5}$ III-V Lab, a joint Laboratory of Alcatel Lucent Bell Labs and Thales Research \& Technology and CEA-LETI, Route \\ de Nozay, 91460 Marcoussis, France \\ cychang@gatech.edu
}

\begin{abstract}
We present a simultaneous measurement of the route to chaos of the optical intensity and carrier density as the feedback strength is increased in an external cavity semiconductor laser (ECL). The intensity is measured with a high-speed photodetector while the carrier dynamics is obtained through a measurement of the dynamic voltage across the laser diode injection terminals. Both measurements demonstrate a route to chaos that stems from the undamping of the relaxation oscillation and provide confirmation of the predictions of the Lang and Kobayashi model concerning the routes to chaos
\end{abstract}

OCIS codes: (190.3100) Instability and chaos; (250.5960) Semiconductor laser.

In an external cavity laser (ECL) setup, a laser diode is place in front of a mirror and the reflected light is coupled back to the active region of the diode. The high speed and the complexity of an ECL have been widely investigated as a prototypical high-dimensional ultrafast dynamical system. An ECL with high tunability of the parameters is also well suited for a test bed for fundamental studies of nonlinear optics as well as for applications such as light detecting and ranging (LIDAR) [1], optoelectronic oscillator [2], high-speed random number generation [3-5], and reservoir computing [6].

Here, we present a simultaneous measurement of the dynamic optical intensity $I(t)$ and the carrier density of our ECL system. We monitor the time-dependent voltage $V(t)$ across the injection terminals of the laser diode under constant-current $J$ injection as a surrogate for the latter. We verify that the dynamics exhibited by $V(t)$ is indeed chaotic and of comparable dynamical complexity as that of $I(t)$ by means of the largest Lyapunov-exponent (LLE) analysis. The basis for our observation is that for small signals, $V(t)$ is proportional to the inversion $N(t)$ in the gain medium, as was pointed out in Refs. 7-9. The dynamics of $N(t)$ and $I(t)$, in turn, are closely linked, as is understood, for example, on the basis of the Lang-Kobayashi equations [10].

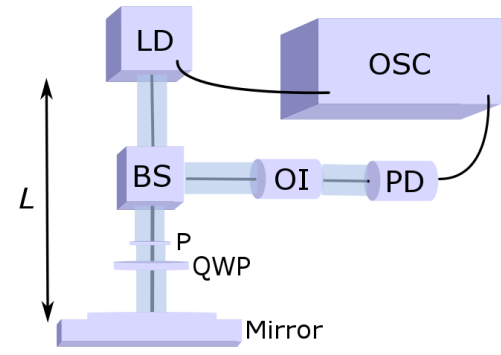

Fig. 1. Experimental setup. LD: laser diode, OSC: oscilloscope, BS: beam splitter, OI: optical isolator, PD: photodiode, P: polarizer, QWP: quarter-wave plate. 
The experimental setup is shown in Fig. 1. The single longitudinal-mode edge-emitting InGaAsP multi-quantumwell DFB LD emits at $1550 \mathrm{~nm}$ with free-running threshold current $J_{t h}=29.8 \mathrm{~mA}$. The experimental feedback strength $\eta$ is determined by the relative angle between the polarizer and the quarter-wave plate (QWP), whereas $\eta=1$ corresponds to maximum feedback strength $\eta_{\max }(\sim 16 \%$ of the optical power that is coupled back onto the collimating lens). $I(t)$ and $V(t)$ are both captured by a real-time oscilloscope whose cut-off frequency is $12 \mathrm{GHz}$.
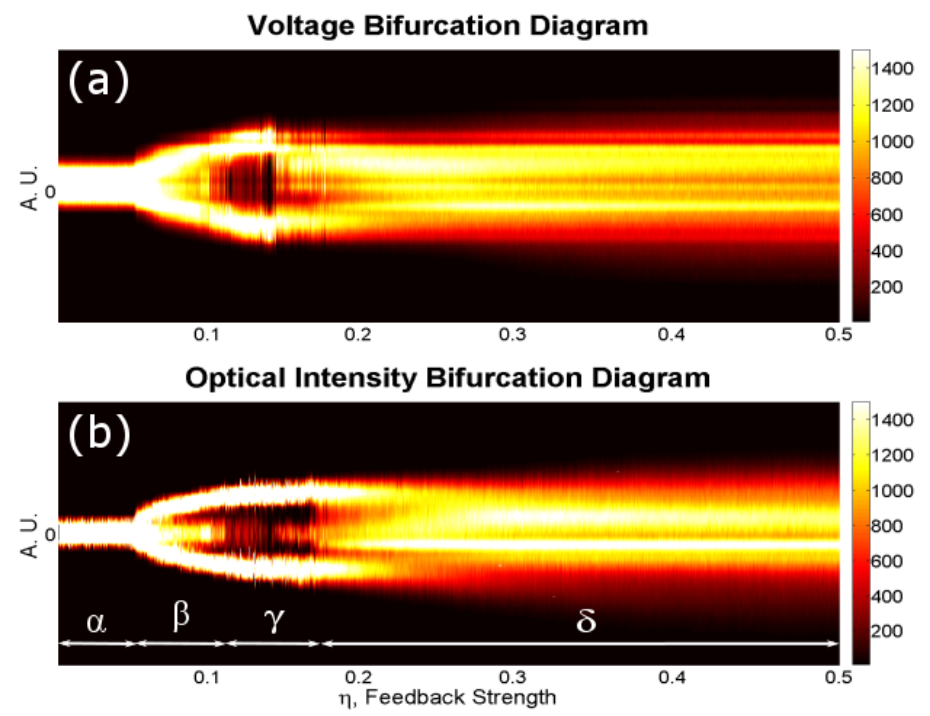

Fig. 2. (a) Voltage $V(t)$ and (b) optical intensity $I(t)$ bifurcation diagrams (BDs) for $J=70.12 \mathrm{~mA}$ and $L=42 \mathrm{~cm}$ as feedback strength $\eta$ is increased.

Figure 2 shows the simultaneously measured bifurcation diagrams (BDs) for $V(t)$ and $I(t)$ which indicate the dynamical regime of the ECL [12]. Each BD is obtained by plotting the density of local extrema of the corresponding time series as the $\eta$ is increased. In the BDs, the density is high in white but low in black. BDs have been obtained recently for the optical $I(t)$ alone [11], but this is the first time the sequence of bifurcations of an ECL is observed simultaneously for both variables.

In conclusion, we have demonstrated that voltage $V(t)$ and optical intensity $I(t)$ have similar characteristics which allow us to investigate various routes to chaos. Both signals experience torus and period-doubling bifurcation, periodicity windows, and intermittent behavior which are experimental evidence of high-dimensional dynamics.

\section{References}

1. F. Y. Lin and J. M. Liu, IEEE J. Quantum Electron. 40, 815 (2004).

2. L. Maleki, Nat. Photon. 5, 728 (2011).

3. A. Uchida, K. Amano, M. Inoue, K. Hirano, S. Naito, H. Someya, I. Oowada, T. Kurashige, M. Shiki, S. Yoshimori, K. Yoshimura, and P. Davis, Nat. Photon. 2, 728 (2008).

4. K. Hirano, T. Yamazako, S. Morikatsu, H. Okumura, H. Aida, A. Uchida, S. Yoshimori, K. Yoshimura, T. Harayama, and P. Davis, Opt. Exp. 18, 5512 (2010).

5. N. Li, B. Kim, V. N. Chizhevsky, A. Locquet, M. Bloch, D. S. Citrin, and W. Pan, Opt. Expr. 22, 6634 (2014).

6. L. Appeltant, M. C. Soriano, G. Van der Sande, J. Danckaert, S. Massar, J. Dambre, B. Schrauwen, C. R. Mirasso and I. Fischer, Nature Commun., 2, 468 (2011).

7. R. F. Kazarinov and R. Suris, Sov. Phys.-JETP 39, 522 (1974).

8. A. A. Sahai, B. Kim, D. Choi, A. Locquet, and D. S. Citrin, Opt. Lett. 39, 5630 (2014).

9. W. Ray, W. Lam, P. N. Guzdar, and R. Roy, Phys. Rev. E 73, 026219 (2006).

10. R. Lang and K. Kobayashi, IEEE J. Quantum Electron. 16, 347 (1980).

11. B. Kim, N. Li, A. Locquet, and D. S. Citrin, Opt. Exp. 22, 2348(2014).

12. B. Kim, A. Locquet, D. Choi, and D. S. Citrin, Phys. Rev. A 91, 061802(R) (2015). 